\title{
A Study of Sodium and Potassium Ion Disturbances in Malaria at Mangalore, Karnataka, India
}

\author{
Roshan Naik ${ }^{1}$, E.V.S. Maben ${ }^{2}$ \\ ${ }^{1}$ Postgraduate (Junior Resident), Department of General Medicine, A.J. Institute of Medical Sciences and Research Centre, \\ Mangalore, ${ }^{2}$ Professor and HOD, Department of General Medicine, A.J. Institute of medical sciences and Research Centre, \\ Mangalore, India
}

Corresponding author: Dr. Roshan Naik, Postgraduate (Junior Resident), Department of General Medicine, A.J. Institute of Medical Sciences and Research Centre, NH-66, Kuntikana, Mangalore - 575004. Karnataka, India.

DOI: http://dx.doi.org/10.21276/ijcmsr.2019.4.4.46

How to cite this article: Roshan Naik, E.V.S. Maben. A study of sodium and potassium lon disturbances in malaria at Mangalore, Karnataka, India. nternational Journal of Contemporary Medicine Surgery and Radiology. 2019;4(4):D191-D194.

\section{A B S T R A C T}

Introduction: Malaria is among the most widespread infectious diseases known to humans. Electrolyte imbalance is one of the major consequences of malaria with studies reporting change in the serum sodium and potassium concentration. Since electrolyte imbalance is relatively unexplored complication. The present study was conducted to determine distribution of sodium and potassium level in patients with different type of malaria and to determine the most common serum abnormality. Material and methods: This was an observation cross sectional. The diagnosis of malaria was made with examination of the peripheral smears and malarial antigen detection rapid card test. 114 diagnosed cases of malaria were enrolled in the study. Evaluation of serum electrolyte (Sodium, Potassium) was done. The data was collected in pre -prepared proforma and then transferred to a master chart for analysis. ANOVA test or Kruskal-Wallis non parametric ANOVA test was used. Level of significance was considered as $\mathrm{P} \leq 0.05$. Software used for analysis was Graph Pad Prism.

Results: Plasmodium Vivax was the most common type of malarial infection. The average serum sodium and potassium among study participants was $132.41 \pm 3.11 \mathrm{mEq} / \mathrm{L}$ and $3.67 \pm 0.40 \mathrm{mEq} / \mathrm{L}$ respectively. Average serum sodium level was less in the patients affected with Plasmodium falciparum infection. Mild hypokalemia was seen in 50 (43.85\%) patients whereas moderate was seen in $1.75 \%$ patients.

Conclusion: Hyponatremia was the most common electrolyte abnormality followed by hypokalemia seen in malaria infection. Electrolyte abnormalities is a common feature in malarial infection and should be monitored regularly.

Keywords: Malaria, Plasmodium Falciparum, Plasmodium Vivax, Hyponatremia, Hypokalemia.

\section{INTRODUCTION}

Malaria is among the most widespread infectious diseases known to humans. It has affected peoples from more than 100 countries and has caused havoc in the countries health management. Its prevalence is highest in Africa and Southeast Asia. ${ }^{1}$ Approximately 300-500 million cases of morbidity and 2-3 million cases of mortality were reported annually due to malaria. ${ }^{2}$

According to the World malaria report, released in November 2018, there were 219 million cases of malaria in 2017, up from 217 million cases in 2016. The estimated number of malaria deaths stood at 435,000 in 2017, a similar number to the previous year. ${ }^{3}$ The World Health Organisation (WHO) African Region continues to carry a disproportionately high share of the global malaria burden. In 2017, the region was home to $92 \%$ of malaria cases and $93 \%$ of malaria deaths. In 2017, 5 countries accounted for nearly half of all malaria cases worldwide: Nigeria (25\%), 3 the Democratic Republic of the Congo (11\%), Mozambique (5\%), India (4\%) and Uganda (4\%). ${ }^{3}$
As per the National Vector Borne Disease Control Program (NVBDCP) incidence records, in most of India, the annual parasite incidence (API) was $<2$, whereas 2-5 API was in scattered regions, and regions with $>5$ API were scattered in the states of Rajasthan, Karnataka, Southern Madhya Pradesh, Chhattisgarh, Gujarat, Jharkhand, Goa and Orissa and in northeastern states. ${ }^{4}$

Malaria is a mosquito borne disease and it is an endemic disease in Mangalore, Karnataka state. Malaria is caused by unicellular eukaryotic protozoan parasites of the Plasmodium spp. Five species have been dentified to have the capacity of infecting humans these are P.falciparum, P.vivax, P.ovale, P. malariae and P.knowlesi. Various geographical, ethnical and wide dispersion of nine anopheles vector transmitting three plasmodia vector, namely, P. falciparum, P. vivax, and P. malariae, resulted in complex epidemiology of malaria in India. ${ }^{4}$

Following infection by the parasite, the clinical features start from day 8 to 25 after infection. Clinical features are fever, headache, vomiting, hemolytic anemia, jaundice etc. But, malaria, especially falciparum malaria, can cause various 
complications involving various systems of the body.

Sodium $(\mathrm{Na})$, an extracellular fluid cation, regulates the normal distribution of water and osmotic pressure in various body fluids. Disturbance of normal serum sodium level is associated with various health related outcomes. ${ }^{1}$ Potassium $(\mathrm{K})$ is a crucial electrolyte for normal functioning of all body cells, tissues and organs. It helps in maintaining blood $\mathrm{pH}$ and water levels in the body. It is particularly important in skeletal and smooth muscle contraction. ${ }^{1}$ Minor changes in potassium level can cause weakness, fatigue and rapid heart beat. Therefore, its balance is very important for the normal physiology of human body. ${ }^{1}$

Electrolyte disturbances Hyponatremia, hypokalemia are commonly seen in malarial infection. ${ }^{5}$ These electrolyte disturbance acts as an ndicator for the severity of disease. ${ }^{6}$ The pathophysiology of the hyponatremia in malaria remains unclear, but several studies have suggested that an increased secretion of vasopressin, plays an important role. ${ }^{7}$ The reduction in K level was primarily due to loss of 75-80\% potassium by host cell during the malaria attack. ${ }^{8}$

Hypokalemia is considered as a correction of acidosis in malaria. ${ }^{9}$ Salt depletion through prolonged sweating or dilution from administration of intravenous dextrose solutions contribute also adds to the electrolyte imbalance.

Since the prevelance of malaria is high in India and electrolyte imbalance is a common yet unexplored complication associated with it. The present study was conducted to determine distribution of sodium and potassium level in patients with different type of malaria and to determine the most common serum abnormality.

\section{MATERIAL AND METHODS}

This was a Single centric, observational, cross sectional study ,conducted in department of general medicine at A.J.institute of medical sciences and research centre,mangalore between October 2017 to September 2019. After obtaining institutional Ethical Committee approval and written

\begin{tabular}{|l|c|}
\hline Serum & Average ( $\mathrm{mEq} / \mathrm{L})$ \\
\hline Serum sodium & $132.41 \pm 3.11$ \\
\hline Serum potassium & $3.67 \pm 0.40$ \\
\hline \multicolumn{2}{|c|}{ Table-1: Average serum sodium and potassium among en- } \\
rolled patients.
\end{tabular}

informed consent from patients, Total of 114 inpatients with more than 18years of age diagnosis of malaria either by peripheral smear for malarial parasite or malaria antigen detection rapid card test, willing to participate in the study. Patients with pseudo hyponatremia, pseudo hypokalemia and who needs intervention in ICU were excluded from the study .

Before their enrolment in the study, the nature and purpose of the study were explained to all the participants. The diagnosis of malaria was made after the examination of the peripheral smears (thick and thin) and on the basis of the malarial antigen detection rapid card test. For all the patients, their demographic profile, their complete history with vitals and relevant system examination was recorded in a pre -prepared proforma and they were subjected to the relevant investigations like Complete Blood Count (CBC), Random blood sugar. Evaluation of serum electrolyte (Sodium, Potassium) was done by using a ABBOTTCI 4100 fully automated electrolyte analyser. Normal serum levels of sodium $=135-145 \mathrm{mEq} / \mathrm{L}$ and potassium $=3.5-5.5 \mathrm{mEq} / \mathrm{L}$. Hyponatremia and Hypernatremia is defined as plasma sodium concentration $<135 \mathrm{mEq} / \mathrm{L}$ and $>145 \mathrm{mEq} / \mathrm{L}$ respectively. Hypokalemia and Hyperkalemia is defined as plasma potassium concentration $<3.5 \mathrm{mEq} / \mathrm{L}$ and $>5.5 \mathrm{mEq} / \mathrm{L}$ respectively.

\section{STATISTICAL ANALYSIS}

Data was be collected in Microsoft excel version 2015. Descriptive statistics for quantitative variables was represented as mean +/- SD. Qualitative variables was represented as frequency \& percentages. ANOVA test or Kruskal-Wallis non parametric ANOVA test was used depending on the normality of data. Graphical representations was done wherever applicable. Level of significance was considered as $P \leq 0.05$. Software used for analysis was Graph Pad Prism.

\section{RESULTS}

Total 114 diagnosed cases of malaria were included in the study and were evaluated.

The average age of patients in our study was $31.80 \pm 13.92$ years. Majority of the patients were in age group of 18 to 30 years i.e. $59.49 \%$.

Males were in majority in our study i.e. $68.42 \%$. The male to female ratio was 2.1: 1 . P.Vivax was the most common

\begin{tabular}{|l|c|c|c|c|}
\hline Serum Sodium (mEq/L) & P. Falciparum & P. Vivax & P. Falciparum + P. Vivax & Total \\
\hline$>135$ & 1 & 12 & 0 & 13 \\
\hline $131-135$ & 5 & 58 & 11 & 74 \\
\hline $126-130$ & 2 & 14 & 10 & 26 \\
\hline$<126$ & 1 & 0 & 0 & 1 \\
\hline \multicolumn{2}{|r|}{ Table-2: Serum Sodium level and types of malaria. } \\
\hline
\end{tabular}

\begin{tabular}{|l|c|c|}
\hline Type & $\begin{array}{c}\text { Serum } \\
\text { sodium (mEq/L) }\end{array}$ & $\begin{array}{c}\text { P value (Kruskal-Wallis Test (Nonpara- } \\
\text { metric ANOVA)) }\end{array}$ \\
\hline P. Falciparum & $130.33 \pm 5.56$ & \multirow{2}{*}{0.004} \\
\hline P. Vivax & $133.02 \pm 2.72$ \\
\hline Mixed (P. Falciparum + P. Vivax) & $130.85 \pm 2.35$ & \\
\hline \multicolumn{2}{|c|}{ Table-3: Average Serum sodium among patients with different type of malaria. } \\
\hline
\end{tabular}




\begin{tabular}{|l|c|c|c|c|}
\hline Serum potassium (mEq/L) & P. Falciparum & P. Vivax & P. Falciparum + P. Vivax & Total \\
\hline$>4$ & 2 & 12 & 0 & 14 \\
\hline $3.6-4$ & 2 & 35 & 11 & 48 \\
\hline $3.0-3.5$ & 5 & 35 & 10 & 50 \\
\hline $2.5-2.9$ & 0 & 2 & 0 & 2 \\
\hline$<2.5$ & 0 & 0 & 0 & 0 \\
\hline \multicolumn{2}{|r}{} \\
\hline
\end{tabular}

type of malarial infection in our study population. Mixed infection with p.vivax and p.falciparum was the second most common cause of malaria .

The average serum sodium among study participants was $132.41 \pm 3.11 \mathrm{imEq} / \mathrm{L}$. The average serum potassium among study participants was $3.67 \pm 0.40 \mathrm{mEq} / \mathrm{L}$ as per Table- 1 .

Majority of the patients i.e.74 had mild hyponatremia. While moderate and severe hyponatremia was seen in 26 and 1 patients respectively. Severe hyponatremia was seen in 1 patients with P.falciparum infection as perTable-2.Significant difference ( $P=0.004$ ) was seen in the serum sodium level in three type of malarial infection. Average serum sodium level was less in the patients affected with P.falciparum infection as per Table- 3 . No significant difference $(P=0.71)$ was seen in the serum potassium concentration among patients with different type of malarial infection. Mild hypokalemia was seen in $50(43.85 \%)$ patients whereas moderate was seen in $1.75 \%$ patients Table- 4 .

\section{DISCUSSION}

Malaria is still considered as world's biggest killer, even 105 years after Ross discovered transmission process of malaria through malaria parasites. ${ }^{10}$ The average age of patients enrolled in our study was $31.80 \pm 13.92$ years with majority of the patient's i.e.59.49\% in the age group of 18 to 30 years. Higher incidence of malaria in age $<40$ years but severity of malaria increases as the age progress. ${ }^{11}$ Age, particularly $21-$ 40 years are more vulnerable to malaria. ${ }^{12}$ These finding also correlates with study conducted by S.R. Karlekar et al. ${ }^{13}$ and Sahar S et al. ${ }^{14}$

Male patients were in majority in our study, with male to female ratio of 2.1:1. The higher incidence of malaria in male can be explained by the movement of males in wider areas, more chances of mosquito bites. ${ }^{15}$ The above results were similar to study conducted by Gurjeet singh. ${ }^{12}$ Karlekar SR et al. ${ }^{13}$

In our study, the incidence of infection due to p.vivax was.73.68\%, followed by mixed infection $18.42 \%$, p.falciparum i.e.7.89\%. This was somewhat in accordance with the study conducted in central India. ${ }^{12,16,17}$ The above result thus indicates that $P$. vivax is the most widespread infection in India.

Electrolyte imbalance, which is considered as consequence of malaria with unknown pathophysiology, indicates severity of infection. Results of our study shows, malaria caused decrease in both sodium and potassium concentration in the body. The average serum sodium was $132.41 \pm 3.11 \mathrm{mEq} / \mathrm{L}$, significant difference was seen in the serum sodium among with different malarial type, those with p.falciparum was had less serum sodium as compared to those infected with p.vivax and mixed infection. Jasani JH.6 also reported similar findings with average serum sodium of $127.76 \mathrm{mEq} / \mathrm{L}$ in $\mathrm{p}$. falciparum and $132.37 \mathrm{mEq} / \mathrm{L}$ in p.vivax patients. in contrast, Asima Rani ${ }^{1}$ reported no significant difference in the serum sodium concentration in p.falciparum and p.vivax, suggested that the hyponatremia merely reflected the effects of the severity of the disease. ${ }^{6}$

The current study also classifies severity of hyponatremia into mild (130-134mEq/L), moderate (125-129 mEq/L) and severe (less than $126 \mathrm{mEq} / \mathrm{L})$, majority (64.91\%) of patients had mild hyponatremia, moderate to severe cases were seen in $22.80 \%$ and $0.80 \%$ patients and severe hyponatremia was seen only in 1 patient in p.falciparum group. This was in similar lines to study conducted by T.N. Dubey 18, where severe malaria was seen mainly in patients with p.falciparum malaria. The incidence of severe hyponatremia was seen in 7 patient $(7 \%)$ of which 6 were p. falciparum and 1 with mixed severe infection. Jasmin H. et al. ${ }^{6}$ in their study showed 64 cases of severe malaria had hyponatremia compared to 22 cases of non severe malaria , out of 64 cases 50 were suffering from severe p. falciparum.

In our study there was no difference $(\mathrm{P}=0.71)$ in the serum potassium level in patients infected with p.falciparum and p.vivax. Mild and moderate hypokalemia was seen in $43.85 \%$ and $1.75 \%$ patients respectively. Results similar to our study was seen in study conducted by Rani Asima. ${ }^{1}$ In contrast Jasani JH. ${ }^{6}$ reported significantly lower hypokalemia in patients with p.falciparum than those with p.vivax.

Few studies have reported hyperkalemia. Acute renal failure and metabolic acidosis were related to increase in potassium level in study conducted by T.N. Dubey. ${ }^{18}$ Das et.al. Maitland et al also reported hyperkalemia. ${ }^{19,20}$

Overall, results of our study and other available in literature suggests hyponatremia and hypokalemia are common in malaria infection. Since this study was aimed to study electrolyte imbalance, the most common electrolyte imbalance seen was hyponatremia followed by hypokalemia. The limitations of the study was that this was a single centric study, the results obtained here may not be applicable to large group of patients and hence need a large scale randomised control trial to validate the results. The study focused on evaluating the two most commonly disturbed electrolyte i.e. sodium and potassium, analyzing complete calcium and magnesium would have given complete picture of electrolyte disturbance in malaria.

Our study thus recommends that serum electrolytes should be estimated in the malaria patients of all the age groups. Electrolyte imbalance should be treated along the disease, to prevent the complications which might result from electrolyte depletion. The precise patho physiological mechanisms of the hyponatremia in malaria need to be further studied. 


\section{CONCLUSION}

Hyponatremia was the most common electrolyte abnormality followed by hypokalemia seen in malaria infection. Electrolyte abnormalities is a common feature in malarial infection and should be monitored regularly.

\section{REFERENCES}

1. Rani A, Akhtar S, Nawaz SK, Irfan S, Azam S, Arshad M. Electrolyte Disturbance and the Type of Malarial infection. ran J Public Health. 2015;44(11):1492-1497.

2. Mishra SK, Mohapatra S, Mohanty Patel NC, Mohapatra DN. Acute renal failure in falciparum malaria. Indian Academy of Clinical Medicine 2002; 3(4): 141-47.

3. WHO. Malaria. Available at https://www.who.int/ news-room/fact-sheets/detail/malaria. Cited on 3rd September 2019.

4. Kumar A, Valecha N, Jain T, Dash AP. Burden of Malaria in India: Retrospective and Prospective View. Am J Trop Med Hyg. 2007;77(6):69-78.

5. Sitprija V. Altered fluid, electrolyte and mineral status in tropical disease, with an emphasis on malaria and leptospirosis. Nat Clin PractNephrol. 2008;4(2):91-101.

6. Jasani JH, Sancheti SM, Gheewala BS, Bhuva KV, Doctor VS, Vacchani AB.et.al. Association of the Electrolyte Disturbances $(\mathrm{Na}+\mathrm{K}+)$ with Type and Severity of Malarial Parasitic infection. J ClinDiagn Res. 2012;6(4):678-681.

7. Sowunmi A, Newton CR, Waruiru C, Lightman S, Dunger DB. Arginine and vasopressin secretion in Kenyan children with severe malaria. J Trop. Pediatr 2000;46(5):195-199.

8. ilkekpeazu EJ , Neboh EE , Aguchime NC, Maduka C, Anyanwu EG. Malaria parasitaemia: effect on serum sodium and potassium levels. Biology and Medicine.2010; 2 (2): 20-25

9. Maitland K, Pamba A, Fegan G, Njuguna P, Nadel S, Newton CR.et.al. Pertur-bations in electrolyte levels in Kenyan children with severe malaria complicated by acidosis. Clin nfect Dis. 2005;40(1):9-16.

10. Hemingwaya J, Bates . Malaria: past problems and future prospects.i EMBO Rep. 2003;4(1): 29-S31.

11. Schwartz E, Sadetzki S, Murad H, Raveh D.Age as a Risk Factor for Severe Plasmodium falciparum Malaria in Nonimmune Patients Clinical infectious Diseases. 2001; 33:1774-7.

12. Singh G, Urhekar AD, Maheshwari U, Samant P. Malarial infection: effects on electrolytes parameters. AARJMD.2015; 1(32);211-216

13. Karlekar SR, Deshpande MM, Andrew RJ. Prevalence of Asymptomatic Plasmodium vivax and Plasmodium falciparum nfections in Tribal Population of a Village in Gadchiroli District of Maharashtra State, India. Biological Forum-An International Journal.2012; 4(4): 42-44.

14. Sahar S, Akhtar T, Bilal H, Rana MS. Prevalence of plasmodium falciparum, malarial parasite in Muzaffargarh district, Punjab-Pakistan: a two year study. Pakistan Journal of Science.2012; 64(2): 64-66.

15. Reuben R. Women and Malaria - Special Risks and Appropriate Control Strategy. Social Science and
Medicine, 1993, 37(4):473-480.

16. Gupta NK, Bansal SB, Jain UC, Sahare K. Study of thrombocytopenia in patients of malaria. Trop Parasitol 2013; 3: 58-61.

17. Asma U, Taufiq F, Khan W. Prevalence and Clinical Manifestations of Malaria in Aligarh, India. Korean J Parasitol.2014;52(6): 621-629.

18. Dubey TN, Gupta N. Acid - Base imbalance and Dyselectrolytemia in Malaria. JMSCR. 2017;5(6):23365-71.

19. Das K, Sastry AS, Sahoo AK, Mahapatra SC.AcidBase imbalance and Dyselectrolytemia in Falciparum Malaria.Indian Medical Gazette. 2014; 283-287.

20. Maitland K, Pamba A, Newton CR, Lowe B, Levin M. Hypokalemia in children with severe falciparum malaria. PediatrCrit Care Med. 2004;5(6):81-5.

Source of Support: Nil; Conflict of Interest: None

Submitted: 31-11-2019; Accepted: 25-12-2019; Published online: 31-12-2019 